\section{Fine-tuned Head Weight Estimation in Globe Artichoke (Cynara scolymus L.)}

\author{
Eftal Düzyaman
}

Ege University, Faculty of Agriculture, Department of Horticulture, 35100

Bornova, Izmir, Turkey

\author{
Basak Ünver Düzyaman \\ Özgörkey Frozen Food Processing Company, Torball, Izmir, Turkey
}

Additional index words. head morphology, head structure, methodology

\begin{abstract}
A novel and nondestructive method for head weight estimation in globe artichoke was described. Linear measurements on head height $(h)$ and head diameter $(2 r)$ were performed on head samples of five cultivars and one developed clone having cylindrical, conical or spherical head forms. The measurements on $2 r$ were performed at the base in conical- and cylindrical-headed cultivars, while they were taken equatorially in spherically headed cultivars. Correlation and regression analyses were performed between single true head weights and eight different models $\left[h, r, 2 r, r^{3}, r^{2} h, \pi r^{2} h\right.$ (cylinder volume), ${ }_{1 / 3}^{1} \pi r^{2} h$ (cone volume) and ${ }^{4} / 3 \pi r^{3}$ (sphere volume)]. Since $\pi r^{2} h$ and ${ }^{1 /} / 3 r^{2} h$ are folds of $r^{2} h$, and ${ }^{4} / 3 \pi r^{3}$ of $r^{3} ; r^{2} h$ or $r^{3}$ had completely the same correlation coefficients as their folds, and hence were equally effective in the statistical analyses. Head weights were more precisely estimated when the $r^{2} h$ model (or their folds) was used for cylindrical and conical heads and the $r^{3}$ model (or their folds) for spherical heads then any other model. Coefficients of determination $\left(R^{2}\right)$ explained the highest variability observed for true head weights when the $r^{2} h$ model was used as the independent variable in the regression analysis for cylindrical and conical cultivars $(96.6 \%$ to $97.5 \%)$ and the $r^{3}$ model for the spherical cultivars $(96.6 \%$ to $98.4 \%)$. Even though all correlation coefficients and regression $F$ values were very highly significant $(p<0.001), 81 \%$ of estimated cases had $<\mathbf{1 0 \%}$ deviations when one of the appropriate models were used in comparison to $66 \%$ of the estimated cases having $<10 \%$ deviations when, for example, the $2 r$ model was used, the second most effective model for all types of heads. The agreement between true and estimated head weights was also tested, where the bias was lower for a cultivar-specific model vs. the $2 r$ model. Discussions on applying the results to the selection procedures were made.
\end{abstract}

Estimations of the magnitude of several plant parts are frequently used to numerically interpret commercial crops species. Despite the fact that weight measurements are comparatively fast and precise, they may not always be easy to perform when, for example, a preharvest estimation is required as it is often the case in physiologic, agronomic or genetic studies (Wright et al., 1990; NeSmith, 1991; Jenni et al., 1996). Geometrical measurements such as height and width of fruit, leafs and heads, on the other hand, are quick and easy to perform in out- and indoor condition. Although, they can be of less value unless they estimate the plant part of interest with high confidence. For instance, various combinations of leaf length and width are used to estimate leaf lamina area (NeSmith, 1991). Similarly, Jenni et al. (1996) estimated the volume of growing ovaries of eastern-type muskmelon from measurements of polar and equatorial diameters. However, yield prediction based on numerical estimations of edible plant parts is given less attention, possibly due to the high degree of interfering factors which limits its practical value (Wright et al., 1990; Lopez-Anido et al., 1998; Marini, 2001).

Amultitude of head forms such as cylindrical, circular cone, ellipsoidal, ovoid and spherical forms occur within the genetic resources

Received for publication 9 Aug. 2004. Accepted for publication 19 Oct. 2004.

of the globe artichoke (Basnizki and Zohary, 1994). So far, only limited attention is given to the form of a plant part, hence of artichoke heads, which magnitude (e.g., weight) is to be estimated. We conducted this research to develop an accurate and nondestructive method to estimate single head weights in globe artichoke based on linear measurements. The aim was to facilitate preharvest estimation of head weights which would enable the comparison of the magnitude of the head of different clones for both breeding purposes and physiological studies. Volume formulas of different forms,

Table 1. Head shapes of cultivars in the experiment. which are commonly used geometrical parameters in mathematics and physics, were taken as criteria in estimating the magnitude of artichoke heads with diverse forms.

\section{Materials and Methods}

A collection farm representing all possible artichoke cultivars grown in Turkey, was established at the Ege University, Faculty of Agriculture, Department of Horticulture, Izmir Province, latitude $38^{\circ} 28^{\prime} \mathrm{N}$, longitude $27^{\circ} 15^{\prime} \mathrm{E}$, altitude $25 \mathrm{~m}$ after a collection mission in 2000 . Five cultivars and one developed clone (for convenience called as a cultivar hereafter) with cylindrical, conical and spherical head forms were chosen from the experimental field (Table 1). Two hundred true to type head samples of each cultivar were carefully selected within the collection farm during the harvest period of 2004. To simulate preharvest conditions, all heads were immediately weighted before significant water loss occurred. Care was also given to collect heads at edible stage but at a wide range of sizes (Table 2). Head height $(h)$ was measured from the base to the end of the bracts in all cultivars in contrast to the measurements on head diameter $(2 r)$, which was taken at the base for conical and cylindrical headed cultivars, and equatorially in spherically headed cultivars. This allowed the application of cylindrical $\left(\pi r^{2} h\right)$, conical $\left(1 /{ }_{3} \pi r^{2} h\right)$ and spherical $\left({ }^{4} / r_{3} \pi r^{3}\right)$ volume formula to the obtained data set. The true volume of each head was also taken by water displacement. ANOVA, correlation, regression analyses, standard deviations and the paired-sample $t$ test were made using appropriate subroutines of the SPSS statistical package (version 11.0).

\begin{tabular}{lcc}
\hline Cultivar & Abbreviation & Head shape \\
\hline Bayrampasa & BPS & Cylinder \\
Sakiz & SKZ & Cylinder \\
Kibris Karas1 & KKAR & Circular cone \\
No 6 & NO6 & Circular cone \\
Isi 2165 & ISI & Sphere \\
Kibris Erkenci & KERK & Sphere
\end{tabular}


structure being either dense or lose. Hence, specific gravity values were also underestimated by changing between 0.695 and $0.755 \mathrm{~g} \cdot \mathrm{cm}^{-3}$, being even less then that of water (data not presented). This was possibly due to the hollowcentric structure of the heads, where water did not enter properly during the measurements. A head weight estimation by calculating volume $\left(\mathrm{cm}^{3}\right) \times$ specific gravity $\left(\mathrm{g} \cdot \mathrm{cm}^{-3}\right)=$ weight $(\mathrm{g})$ was therefore useless.

The results of the correlation analysis are presented in Table 4. All correlation coefficients between true head weights and the models (including $h, 2 r$, and $r$ alone) were highly significant at $p<0.001$ level. Completely the same correlations coefficients were obtained when the cylinder formula $\left(\pi r^{2} h\right)$, the circular cone formula $\left(1 / 3 \pi r^{2} h\right)$ or $r^{2} h$ alone was plotted against the head weight. The reason for this is simply the fact that the formulas for circular cone and cylinder are folds of $r^{2} h$. Similar was the case for the sphere formula $\left(4 / 3 \pi r^{3}\right)$ which was a fold of $r^{3}$. This fact allowed a simple replacement of cylindrical and conical models by $r^{2} h$, and the spherical model by $r^{3}$. Correlation coefficients for the $r^{3}$ model in the case of cylindrical and conical heads and the $r^{2} h$ model in the case of spherical heads are calculated but are erroneously since they consider the wrong diameter. However, this was just done

Table 2. True head weights of the cultivars.

\begin{tabular}{lccc}
\hline & \multicolumn{3}{c}{ Head wt $(\mathrm{g})$} \\
\cline { 2 - 4 } Cultivar & Minimum & Maximum & Mean \\
\hline SKZ & 51 & 300 & 163 \\
BPS & 62 & 293 & 169 \\
KKAR & 27 & 240 & 117 \\
NO6 & 38 & 301 & 118 \\
ISI & 67 & 272 & 145 \\
KERK & 50 & 328 & 149 \\
\hline
\end{tabular}

Table 3. Average true and estimated head volumes of the cultivars compared by paired-sample $t$ test.

\begin{tabular}{lccc}
\hline Cultivar & $\begin{array}{c}\text { True } \\
(\mathrm{mL})\end{array}$ & $\begin{array}{c}\text { Estimated } \\
(\mathrm{mL})\end{array}$ & $\begin{array}{c}\text { Deviation } \\
(\%)\end{array}$ \\
\hline BPS & $224.2 \mathrm{~b}$ & $396.3 \mathrm{a}$ & 76.7 \\
SKZ & $221.2 \mathrm{~b}$ & $363.7 \mathrm{a}$ & 64.4 \\
KKAR & $167.8 \mathrm{a}$ & $106.0 \mathrm{~b}$ & -36.8 \\
NO6 & $156.7 \mathrm{a}$ & $99.7 \mathrm{~b}$ & -36.4 \\
ISI & $195.7 \mathrm{~b}$ & $219.6 \mathrm{a}$ & 12.2 \\
KERK & $210.8 \mathrm{~b}$ & $269.3 \mathrm{a}$ & 27.8 \\
\hline
\end{tabular}

Table 4. Correlation coefficients between the models and true head weights.

\begin{tabular}{|c|c|c|c|c|c|c|}
\hline \multirow[b]{3}{*}{ Model } & \multicolumn{6}{|c|}{ True head weights } \\
\hline & \multicolumn{2}{|c|}{ Cylinder } & \multicolumn{2}{|c|}{ Circuar cone } & \multicolumn{2}{|c|}{ Sphere } \\
\hline & BPS & SKZ & KKAR & NO6 & ISI & KERK \\
\hline $\bar{h}$ & 0.769 & 0.836 & 0.835 & 0.805 & 0.510 & 0.625 \\
\hline $2 r($ or $r)$ & 0.949 & 0.965 & 0.969 & 0.926 & 0.968 & 0.976 \\
\hline$r^{2} h$ or $\pi r^{2} h$ or ${ }^{1 /} \pi r^{2} h$ & 0.987 & 0.985 & 0.987 & 0.983 & 0.918 & 0.965 \\
\hline$r^{3}$ or $4 / 3 \pi r^{3}$ & 0.955 & 0.965 & 0.976 & 0.934 & 0.983 & 0.992 \\
\hline
\end{tabular}

Table 5. Coefficients of determination $\left(\mathrm{R}^{2}\right)$ between the models and true head weights.

\begin{tabular}{|c|c|c|c|c|c|c|}
\hline \multirow[b]{3}{*}{ Model } & \multicolumn{6}{|c|}{ True head weights } \\
\hline & \multicolumn{2}{|c|}{ Cylinder } & \multicolumn{2}{|c|}{ Circuar cone } & \multicolumn{2}{|c|}{ Sphere } \\
\hline & BPS & $\mathrm{SKZ}$ & KKAR & NO6 & ISI & KERK \\
\hline $\bar{h}$ & 0.590 & 0.697 & 0.696 & 0.647 & 0.257 & 0.387 \\
\hline $2 r($ or $r)$ & 0.912 & 0.931 & 0.938 & 0.857 & 0.937 & 0.951 \\
\hline$r^{2} h$ or $\pi r^{2} h$ or ${ }^{1 /} \pi r^{2} h$ & 0.975 & 0.970 & 0.975 & 0.966 & 0.841 & 0.930 \\
\hline$r^{3}$ or $4 / 2 \pi r^{3}$ & 0.911 & 0.931 & 0.951 & 0.872 & 0.966 & 0.984 \\
\hline Combined & \multicolumn{2}{|c|}{0.969} & \multicolumn{2}{|c|}{0.963} & \multicolumn{2}{|c|}{$\begin{array}{lll}0.500 & 0.953 & \end{array}$} \\
\hline
\end{tabular}

types, respectively, which were still higher then other models.

To have a better imagination of the dispersion of single cases, biplots for $r^{2} h \times$ true head weight and $2 r \times$ true head weight are compared in Fig. 1 for the cultivar BPS as a sample. The reason why $2 r$ was chosen for comparison was the fact that in general it had the second highest correlation coefficients or $R^{2}$ values after the cultivar-specific $r^{2} h$ or $r^{3}$ models. Despite that the explained proportion of variation for true head weight is very high (91.2\%) in $2 r$ $\times$ true head weight and close to that of $r^{2} h \times$ true head weight $(97.5 \%)$, a more linear view was obtained for $r^{2} h \times$ true head weight when compared to $2 r \times$ true head weight.

High correlation coefficients are often misinterpreted as an indication of good agreement between the true and the estimated value. As reviewed by Marini (2001), the degree of agreement between the estimated and true values can be evaluated by calculating the bias, estimated by the mean of the differences (d) and the standard deviation of the differences (SD) between the true values and the estimated values (Table 6). The limits of agreement are then defined as $\mathrm{d} \pm 2$ SD where most differences are expected to lie. The $2 r$ model was taken as a base for comparison, since it had the highest correlation coefficient and $R^{2}$ values after the cultivar-specific $r^{2} h$ or $r^{3}$ models. Higher values for standard deviations of differences were evident the $2 r$ model as compared to the $r^{2} h$ (for cylindrical and conical cultivars) or $r^{3}$ (for spherical cultivars) models. The limits of agreements are inevitably higher in the $2 r$ model than in the $r^{2} h$ or $r^{3}$ models since it consider a greater range of differences $(\mathrm{d} \pm$ $2 \mathrm{SD}$ ) with standard deviation of the differences between the estimated and true mean head weight as the main determining factor. However, cases within the limits of agreement were still slightly lower in the $2 r$ model then in the cultivar specific $r^{2} h$ or $r^{3}$ models.

Additionally, percent deviations in estimated head weights from the true head weights calculated from the regression equations of i) the best fitted model $\left(r^{2} h\right.$ for cylindrical and conical and $r^{3}$ for spherical cultivars) in comparison to ii) a less functional model $(2 r)$ are presented in Fig. 2. To have a more comparative view of both models, all single cases were ranked from small to great for each model separately. Since similar headed cultivars showed similar deviations data were bulked at the basis of head forms. For instance, when the $r^{2} h$ model was used in the regression analysis with cylindrical and conical headed cultivars, deviations were less then $10 \%$ in $85 \%$ of the cylindrical and $75 \%$ of the conical heads, while only $3 \%$ and $4 \%$ of the heads, respectively had them more then $20 \%$. However, when the $2 r$ model was used only $63 \%$ and $61 \%$ of the head samples had $<10 \%$ deviation, while $11 \%$ and $15 \%$ had them $>20 \%$ for cylindrical and conical cultivars, respectively. Similar were the figures for the bulked data of spherical headed cultivars. In general, cylindrical and spherical cultivars tend to be underestimated and conical cultivars overestimated when the $2 r$ model was used. 
Regression equations presented in Table 7 are given for the best fitted models only. Differences in regression slopes $\left(b_{1}\right)$ were low for all cultivars having similar head types, while the difference in the intercept $\left(b_{0}\right)$ was comparatively large only between the spherically headed cultivars ISI and KERK. The homogeneity of slopes for BPS vs. SKZ, KKAR vs. NO6, and ISI vs. KERK was tested to elucidate whether a combined model was appropriate for similar headed cultivars. This was done by developing a model containing

Fig. 1. A sample of distribution of single cases in biplots between true head weight and the $r^{2} h$ (a) or the $2 r$ (b) model in the cylindrical headed cultivars BPS.
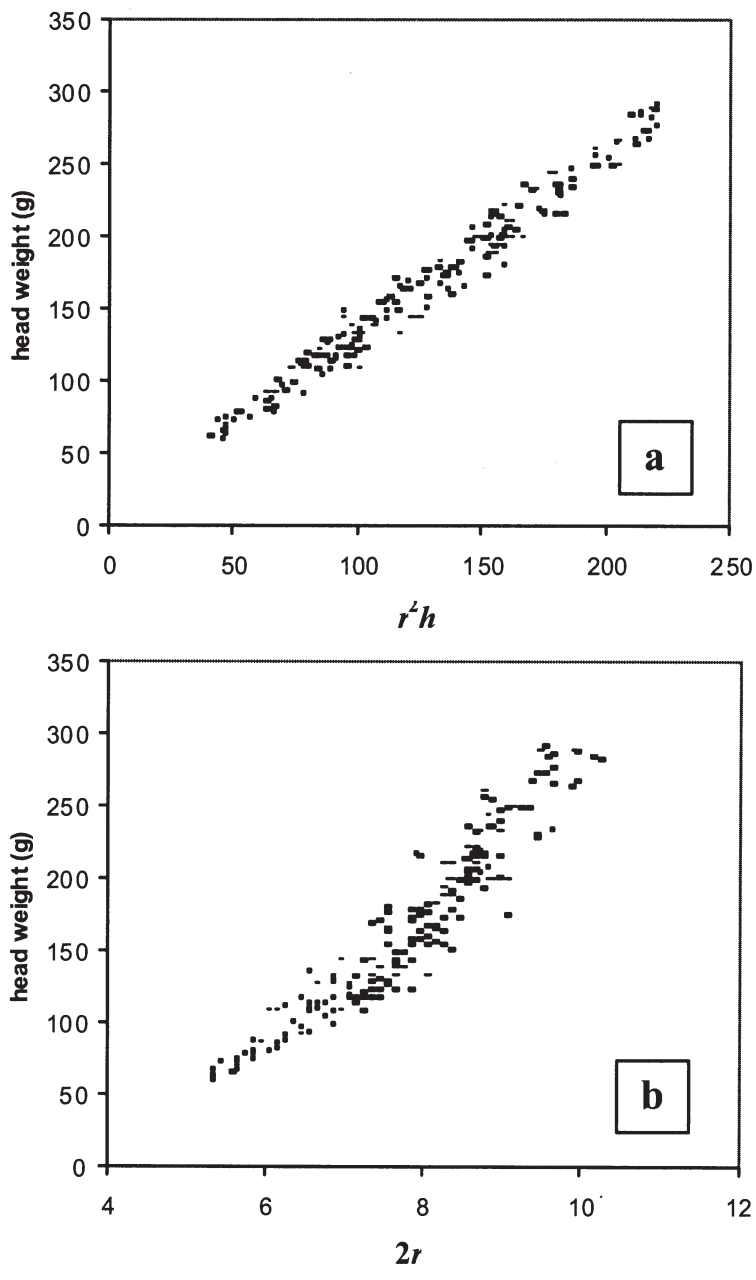

cultivar and $\mathrm{x}$ as qualitative variables and the interaction term of cultivar $\times \mathrm{x}$. The null hypothesis was that the slopes were equal. If the slopes are equal then a combined model may be used for similar headed cultivars. A significant interaction term would reject the null hypothesis and there would be a need for individual models instead of the combined model. However, it was found that the interaction term was neither significant in all tree cases, which accepts the null hypothesis that the slopes are equal and enables the use of the combined regression equations for similar headed cultivars.

Similar to our study, linear measurements are often combined in estimation studies, e.g., a leaf length $\times$ leaf width combination instead leaf length alone was suggested as a far better interpretation of the leaf area in various cultivars of Vaccinium ashei (NeSmith, 1991).

Screening head weights of large numbers of artichokes clones is often necessary in large scale breeding programs even when head weight is not the only and primary trait of interest (Lopez-Anido et al., 1998). Since head weight is only being determined after harvest, labeling and weighting each head separately creates additional costs and labor and sets practical limits to the magnitude of the clonal selection program. It is not uncommon for most breeders to act with rough estimates in such cases. The head weight estimation method described here is based on linear measurements which can be carried out easily in outdoor conditions. Hence, it would simplify the comparison of diverse head forms often present in germplasm evaluation programs (Lopez-Anido et al., 1998), simply by calculating the dependent variable y from one of the models $r^{2} h$ or $r^{3}$. Moreover, taking the basal $2 r$ in cylindrical and conical headed individuals (and possibly in ovoid heads), and the equatorial $2 r$ in spherical

Table 6. Number of cases within the limits of agreement for each model in the regression equations.

\begin{tabular}{|c|c|c|c|c|c|c|c|}
\hline \multirow[b]{2}{*}{ Cultivar } & \multirow[b]{2}{*}{ Model } & \multirow{2}{*}{$\begin{array}{c}\text { Mean of } \\
\text { differences } \\
(d)^{z}\end{array}$} & \multirow{2}{*}{$\begin{array}{c}\text { SD } \\
\text { of } \\
\text { differences }\end{array}$} & \multicolumn{2}{|c|}{ Limits of agreement } & \multirow{2}{*}{$\begin{array}{l}\text { Cases within the } \\
\text { limits of agreement } \\
\text { (no.) }\end{array}$} & \multirow{2}{*}{$\begin{array}{c}\text { Cases within the } \\
\text { limits of agreement } \\
(\%)\end{array}$} \\
\hline & & & & $\mathrm{d}+2 \mathrm{SD}$ & $\overline{d-2 S D}$ & & \\
\hline \multirow[t]{2}{*}{$\overline{\mathrm{BPS}}$} & $r^{2} h$ & 0.030 & 9.53 & 19.10 & -19.04 & 191 & 95.5 \\
\hline & $2 r$ & 0.030 & 18.97 & 37.96 & -37.90 & 190 & 95.0 \\
\hline \multirow[t]{2}{*}{ SKZ } & $r^{2} h$ & -0.001 & 10.28 & 20.56 & -20.56 & 194 & 97.0 \\
\hline & $2 r$ & -0.001 & 15.59 & 31.17 & -31.17 & 189 & 94.5 \\
\hline \multirow[t]{2}{*}{ KKAR } & $r^{2} h$ & -0.531 & 8.18 & 15.83 & -16.89 & 197 & 98.5 \\
\hline & $2 r$ & 0.000 & 12.91 & 25.83 & -25.83 & 189 & 94.5 \\
\hline \multirow[t]{2}{*}{ NO6 } & $r^{2} h$ & 0.000 & 9.60 & 19.20 & -19.20 & 191 & 95.5 \\
\hline & $2 r$ & 0.000 & 19.80 & 39.59 & -39.59 & 188 & 94.0 \\
\hline \multirow[t]{2}{*}{ ISI } & $r^{3}$ & 0.000 & 8.20 & 16.41 & -16.41 & 199 & 99.5 \\
\hline & $2 r$ & 0.000 & 11.26 & 22.52 & -22.52 & 193 & 96.5 \\
\hline \multirow[t]{2}{*}{ KERK } & $r^{3}$ & -3.000 & 7.79 & 12.57 & -18.57 & 198 & 99.0 \\
\hline & $2 r$ & 0.000 & 13.77 & 27.54 & -27.54 & 191 & 95.5 \\
\hline
\end{tabular}

${ }^{2}$ Between the true head weights and the estimated head weights. 

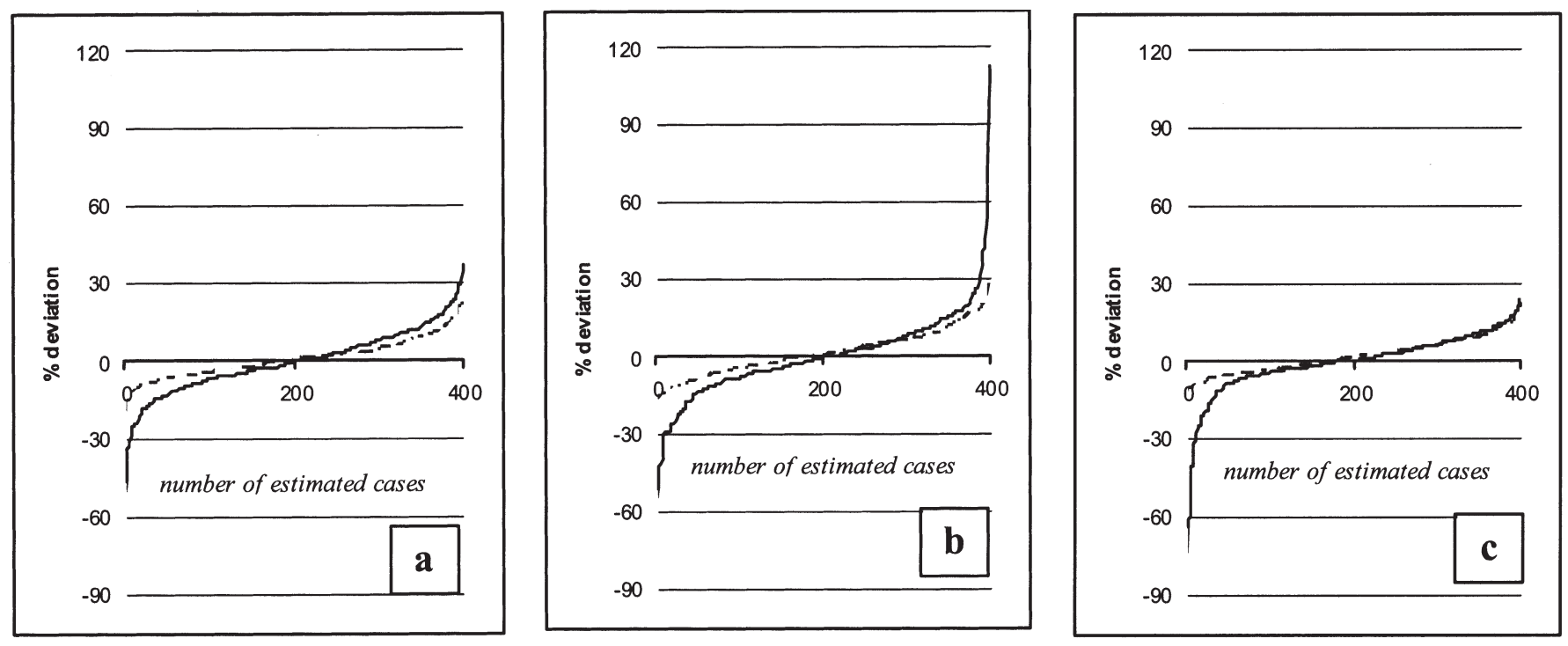

Fig. 2. Percent deviations in estimated head weights from true head weights (y axis) in the regression analyses for 400 heads (x axis). Head weights are either estimated from the regression equation using $2 r$ (continuous line) the second most fitted model for all types of heads or from the regression equations using $r^{2} h$ for cylindrical (a) and conical (b) headed cultivars and $r^{3}$ for spherical (c) headed cultivars (dashed line).

Table 7. Regression equations between head weight (y) and best fitted model (x).

\begin{tabular}{llc}
\hline Cultivar & $\begin{array}{l}\text { Equation } \\
\left(\mathrm{y}=b_{0}+b_{1} \mathrm{x}\right)\end{array}$ & Model \\
\hline BPS & $\mathrm{y}=13.136+1.235 \mathrm{x}$ & $r^{2} h$ \\
SKZ & $\mathrm{y}=13.906+1.287 \mathrm{x}$ & $r^{2} h$ \\
Combined & $\mathrm{y}=14.544+1.251 \mathrm{x}$ & $r^{2} h$ \\
KKAR & $\mathrm{y}=8.057+1.073 \mathrm{x}$ & $r^{2} h$ \\
NO6 & $\mathrm{y}=8.588+1.153 \mathrm{x}$ & $r^{2} h$ \\
Combined & $\mathrm{y}=9.036+1.104 \mathrm{x}$ & $r^{2} h$ \\
ISI & $\mathrm{y}=49.467+1.828 \mathrm{x}$ & $r^{3}$ \\
KERK & $\mathrm{y}=36.160+1.750 \mathrm{x}$ & $r^{3}$ \\
Combined & $\mathrm{y}=46.627+1.719 \mathrm{x}$ & $r^{3}$ \\
\hline
\end{tabular}

headed individuals (and possibly in ellipsoidal heads) is more feasible in terms of conducting the measurements. The nondestructiveness of the method may also serve in physiological studies where repeated measurements are needed. However, it can also be helpful in estimating maximum and minimum values of the same head, which in turn would allow the comparison of mean head values among different germplasm resources. This can especially be of importance in comparisons between early and late clones where the mean of head weights do not coincide as pointed out by Lopez-Anido et al. (1998).
It is known that artichoke heads deviate from their original form when reaching maturity (Mauromicale and Raccuia, 2000), which could increase the error in late measurements. For instance, Jenni et al. (1996) suggested corrections, when using a model to estimate volume of growing ovaries of eastern-type muskmelon, originally developed to predict the volume of mature fruit. However, since our equations are based on a wide range of head sizes, estimations may be less erroneous provided that questioned heads are at edible stage.

Number and weight of artichoke heads are traits having the highest association with final yield (Lopez-Anido et al., 1998), which raises the possibility of predicting and comparing yield of single clones in the experimental field. However, the environmental interference should be considered when translating single head weight estimations, into comprehensive yield predictions (Wright et al., 1990). Hence, a sampling within the edible parts of a plant to estimate fruit weight (and possibly head weight) is also associated with high error, which can be larger than many agronomists would accept (Marini, 2001).

\section{Literature Cited}

Basnizki, J. and D. Zohary. 1994. Breeding of seed planted artichoke. Plant Breed. Rev. 12:253-269.

Jenni, S., K.A. Stewart, G. Bourgeois, and D.C. Cloutier. 1996. Nondestructive volume estimation for growth analysis of eastern-type muskmelon ovaries. HortScience 31:1236.

Lopez-Anido, F.S., I.T. Firpo, S.M. Garcia, and E.L. Cointry. 1998. Estimation of genetic parameters for yield traits in globe artichoke (Cynara scolymus L.). Euphytica 103:61-66.

Marini, R.P. 2001. Estimating mean fruit weight and mean fruit value for apple trees: Comparison of two sampling methods with the true mean. J.Amer. Soc. Hort. Sci. 126:503-510.

Mauromicale, G. and S.A. Raccuia. 2000. Influence of maturation time on some head characteristics of globe artichoke (Cynara scolymus L.). Acta Hort. 533:483-488.

NeSmith, D.S. 1991. Nondestructive leaf area estimation of rabbiteye blueberries. HortScience 26:10.

Seber, G.A.F. 1977. Linear regression analysis. John Wiley \& Sons, New York.

Wright, G.C., J.B. Storey, M.K. Harris, and P.T Sprinz. 1990. Preharvest pecan yield estimation. HortScience 25:698-700. 